\title{
Relationship between oxidative stress and clinical pregnancy in assisted reproductive technology treatment cycles
}

\author{
Yunus Aydin • Orhan Ozatik • Hikmet Hassa • \\ Dilek Ulusoy $\cdot$ Serdal Ogut $\cdot$ Fezan Sahin
}

Received: 30 January 2013 / Accepted: 22 April 2013 /Published online: 11 May 2013

(C) Springer Science+Business Media New York 2013

\begin{abstract}
Purpose To examine the relationship between serum total oxidant (TOS) and antioxidant (TAS) levels and clinical pregnancy in assisted reproductive technology (ART) cycles. Methods Prior to and after oocyte pick-up (OPU) and embryo transfer (ET), blood samples were collected from gynecologically normal females $(n=70)$ who were enrolled for ART solely due to male factor infertility. TAS, TOS levels and oxidative stress indexes (OSI: TOS/TAS) in four
\end{abstract}

Capsule In ART-ICSI cycles, women with lower oxidative stress index (OSI: TOS/TAS) measurements prior to oocyte collection, may have increased probability of clinical pregnancy.

Y. Aydin $(\bowtie)$

Department of Obstetrics and Gynecology,

Eskisehir Osmangazi University School of Medicine,

Eskisehir 26480, Turkey

e-mail: aydin.yunus@yahoo.com

O. Ozatik

Reproductive Medicine Unit, Eskisehir

Osmangazi University School of Medicine,

Eskisehir, Turkey

H. Hassa

Department of Obstetrics and Gynecology,

Reproductive Medicine Unit, Eskisehir Osmangazi University

School of Medicine, Eskisehir, Turkey

D. Ulusoy

Reproductive Medicine Unit, Suleyman Demirel University

School of Medicine, Isparta, Turkey

S. Ogut

Department of Biochemistry, Suleyman Demirel University

School of Medicine, Isparta, Turkey

F. Sahin

Department of Biostatistics, Eskisehir Osmangazi University

School of Medicine, Eskisehir, Turkey phases of treatment cycle (pre and post OPU and ET) between clinically pregnant and non-pregnant patients were compared. Critical cut-off values of significantly different TAS, TOS levels and OSIes for clinical pregnancy were established.

Results The TAS levels in patients with clinical pregnancy were significantly higher in all of the four phases of the cycle. PostOPU and pre-ET TOS levels were significantly higher in clinically pregnant patients. According to OSIes; only the pre-OPU OSI was significantly lower in clinically pregnant patients compared with non-pregnant ones. Moreover, the highest area under the ROC curve (AUC) from the seven different significant measurements [1) pre-OPU OSI, 2) pre-OPU TAS, 3) postOPU TAS, 4) pre-ET TAS, 5) post-ET TAS, 6) post-OPU TOS, 7) pre-ET TOS] was related to pre-OPU OSI.

Conclusions In ART cycles, women with a higher total antioxidant status prior to and after OPU and prior to and after ET have an increased probability of clinical pregnancy. Additionally; OSI before OPU was one of the most important determinants for clinical pregnancy, so the oxidant and antioxidant balance is as important as the antioxidant concentration alone.

Keywords Assisted reproductive technology $\cdot$ Fertility · Oxidative stress $\cdot$ Pregnancy

\section{Introduction}

Considering recent studies, it is believed that the oxidative stress balance plays a significant role in the normal functioning of the female reproductive system as well as many other systems. The development of imbalance between oxidants and antioxidants in the female reproductive system results in oxidative stress (OS) [1]. It was shown that 
reactive oxygen species (ROS), which are markers of oxidative stress, in follicular fluid, are important factors in the development and quality of embryos [7]. In another group of studies, the ROS level in embryo culture medium was reported to negatively affect embryo cleavage and could even help to predict clinical pregnancies [5, 6]. Considering these findings, the ROS level observed as an embryonic metabolic marker in embryo culture medium can be used as one of the criteria for embryo selection, and more clinical pregnancies can be attained by single ET, which can be performed based on this approach [12].

In contrast, oxidative stress markers in peritoneal fluid were higher in unexplained infertile couples, which constitutes one of the major patient groups among infertile individuals [2]. However, in patients with endometriosis, in which the worst results are expected in ART treatment, the antioxidant levels were found to be lower [17]. Today, there is no debate that especially in infertile couples, oxidative stress has an impact on many characteristics, including follicular fluid, embryo culture medium, the endometrial implantation phase and the continuity of the corpus luteum [2]. However, most of the studies on the effect of oxidative stress on female infertility were animal or in vitro studies, and their results were contradictory [3].

Our aim in this study was to examine the relationship between serum total oxidant and antioxidant levels and clinical pregnancy and evaluate this relationship in terms of predicting clinical pregnancy in 70 couples who were admitted to ART treatment solely because of male factor infertility; in these couples, the women were completely normal from a fertility perspective. To our knowledge, no study in the literature has examined the correlation between blood total oxidant and antioxidant levels and clinical pregnancy in women with normal characteristics who were admitted to ART treatment solely due to male factor infertility.

\section{Methods}

This prospective cohort study was conducted between September 2011 and July 2012 at Eskisehir Osmangazi University, Medical Faculty-Center for Reproductive Health, and approved by the Ethical Review Board of the hospital. A total of 70 patients attending the IVF unit of the center for ICSI cycles were recruited according to the following criteria: i) absence of any apparent abnormality of the reproductive system, as revealed by their medical history, clinical examinations and common hormonal tests; ii) absence of any metabolic or endocrine system-associated diseases, such as hyperprolactinemia, thyroid dysfunction, or polycystic ovary syndrome as defined by the Rotterdam criteria; iii) absence of any surgical history regarding the reproductive system; iv) normal ovulatory cycle, with cycle lengths of between 25 and 35 days; v) age of women between 27 and 36 years; vi) expected normal responders, as day 3 basal ultrasonography and a hormone profile revealed the following among all the women: FSH $<10 \mathrm{mIU} / \mathrm{ml}$, estradiol $<40 \mathrm{pg} / \mathrm{ml}$ and antral follicle count (AFC) $>6$; vii) absence of any smoking history in women; and viii) indication for assisted reproductive technology (ART) treatment as exclusively advanced male factor infertility (severe oligoasthenoteratospermia [severe oligospermia $(<5$ million sperm $/ \mathrm{ml}$ ), asthenospermia ( $<5 \%$ progressive motility), or teratospermia $(<4 \%$ normal forms by strict criteria)] according to WHO criteria [21], ix) only couples with sperm can be obtained from ejaculation, so we totally excluded couples who had surgical sperm extraction or aspiration indication due to azoospermia. Patients gave their written informed consent and did not receive any monetary compensation for participating in the study. Only the first cycle of each patient was included in the study.

All women received the long gonadotropin-releasing hormone agonist protocol for controlled ovarian hyper stimulation $(\mathrm{COH})$. To achieve pituitary desensitization, leuprolide acetate (Lucrin ${ }^{\circledR}$; Abbott Laboratories, Istanbul, Turkey) was initiated on day 21 of the previous cycle at a dose of $10 \mathrm{U}$, and it was decreased to $5 \mathrm{U}$ on days $2-3$ of menstrual bleeding. As soon as menstruation began, ovarian stimulation was initiated with 150-250 U/day rec-FSH (Puregon ${ }^{\circledR}$; MSD, Istanbul, Turkey). Recombinant hCG (Ovitrelle ${ }^{\circledR}$, Serono, Istanbul, Turkey) was applied when $\geq 3$ follicles reached $\geq 17 \mathrm{~mm}$, and oocyte pick-up (OPU) was performed under sedation at the 36th hour following hCG. On oocyte retrieval day, sperm was collected into a sterile plastic container from males who refrained from ejaculation for 3-5 days prior to the procedure. Following sperm preparation with a density gradient method, the ICSI procedure was performed using sperm that was selected by embryologists $2-3 \mathrm{~h}$ after OPU. A fertilization (two pronuclei) check was performed on embryos that were followed in cleavage stage medium (G1.3; Vitrolife ${ }^{\circledR}$, Kungsbacka, Sweden) at 16-18 h, and embryos were observed until the 3rd day after OPU. After embryologists performed a control on the 3rd day, one embryo that was grade I according to the ESHRE consensus [4] was selected for transfer. All transfers were performed under ultrasonography, and a soft transfer catheter was used (Wallace Embryo Replacement Catheter, Ref. 1816N; Smiths Medical International Limited, Kent, UK).

Serum hCG levels were measured in all patients on the 14th day following ET. Patients with $>50 \mathrm{IU} / \mathrm{L}$ hCG level were evaluated as pregnancy positive. In patients with a positive initial hCG measurement, a twofold increase was evaluated after $48 \mathrm{~h}$. Transvaginal ultrasonography was performed 3 weeks later in patients who showed an increase, and those with a fetal structure and fetal cardiac activity were considered as clinical pregnancy positive.

Prior to and after oocyte retrieval and prior to and after transfer, 3-5 $\mathrm{ml}$ of fasting blood sample was collected from 
Table 1 The demographic characteristics of the study population

Data are represented with the form of the Mean \pm Standard Deviation (SD)

$B M I$ Body Mass Index; FSH Follicle Stimulating Hormone; LH Luteinizing Hormone; NS Non-significant

$P$-value $<0.05$ was considered statistically significant

\begin{tabular}{lccc}
\hline & $\begin{array}{l}\text { Clinically pregnant } \\
(n=22)\end{array}$ & $\begin{array}{l}\text { Clinically non-pregnant } \\
(n=48)\end{array}$ & $P$ value \\
\hline Female age (years) & $31.5 \pm 3.9$ & $32 \pm 3.2$ & $\mathrm{NS}$ \\
BMI (kg/m²) & $23.4 \pm 2.9$ & $24.2 \pm 2.1$ & $\mathrm{NS}$ \\
Duration of infertility (years) & $3.1 \pm 1.9$ & $2.9 \pm 2.0$ & $\mathrm{NS}$ \\
Basal FSH (mIU/ml) & $7.1 \pm 2.1$ & $8.3 \pm 1.6$ & $\mathrm{NS}$ \\
Basal LH (mIU/ml) & $5.1 \pm 1.2$ & $4.7 \pm 1.4$ & $\mathrm{NS}$ \\
Basal estradiol (pg/ml) & $37 \pm 7$ & $36 \pm 9$ & $\mathrm{NS}$ \\
Basal antral follicle count & $9 \pm 2.1$ & $10 \pm 1.9$ & $\mathrm{NS}$ \\
Number of retrieved oocytes & $15 \pm 4.1$ & $14 \pm 4.9$ & $\mathrm{NS}$ \\
Fertilization rate $(\%)$ & $85 \pm 6.1$ & $79 \pm 4.9$ & $\mathrm{NS}$ \\
Number of day-3 grade I+II embryos & $7 \pm 2.3$ & $6 \pm 3.0$ & $\mathrm{NS}$ \\
Number of transferred embryos & $1 \pm 0$ & $1 \pm 0$ & $\mathrm{NS}$ \\
\hline
\end{tabular}

the forearm between 8 and 10 am in all women and centrifuged at 3,000 rpm, and attained sera were kept at $-80{ }^{\circ} \mathrm{C}$. The total oxidant status (TOS) in the plasma was measured using a novel automated colorimetric measurement method developed by Erel. According to this method, oxidants present in the sample oxidize the ferrousion-odianisidine complex to ferric ion. The results are expressed in terms of micromole hydrogen peroxide equivalent per liter $\left(\mu \mathrm{mol} \mathrm{H}_{2} \mathrm{O}_{2} \mathrm{Eq} / \mathrm{L}\right)$ [10]. The total antioxidant status (TAS) in the plasma was measured using a novel automated colorimetric measurement method developed by Erel [9]. According to this method, the hydroxyl radical, which is produced by the Fenton reaction, reacts with the colorless substrate $o$-dianisidine to produce the bright yellowishbrown dianisyl radical. The assay results are expressed as mmol Trolox Eq/L. TAS and TOS levels in 4 phases of treatment cycle (before OPU, after OPU, before ET, after ET) between clinically pregnant and clinically non-pregnant patients were compared. Critical cut-off values of significantly different TAS and TOS levels for clinical pregnancy were calculated. The oxidative stress indexes (OSI: TOS/TAS) for the evaluation of systemic oxidative stress and antioxidant response in ovarian stimulation and intracytoplasmic sperm injection (ICSI) outcomes were also calculated and critical cut-off value for significant OSIes were found.

This study was statistically analyzed using SPSS IBM 20 and Medcalc 11.3. For all variables, the Shapiro Wilk test was used to assess normality. For normally distributed variables, paired-sample t-tests and independent-sample t-tests were applied, and the mean \pm standard deviation values were presented. Wilcoxon's signed rank test and the Mann-Whitney $U$ test were applied for non-normally distributed variables, and median $(25 \%-75 \%)$ percentiles were presented. Critical cut-off values of significantly different TAS and TOS levels, OSIes for clinical pregnancy

Table 2 TAS and TOS levels before-after oocyte collection, before-after embryo transfer in clinically pregnant and non-pregnant women

\begin{tabular}{llll}
\hline & Clinically pregnant $(n=22)$ & Clinically non-pregnant $(n=48)$ & $P$ value \\
\hline TAS before OPU & $1,70(1,55-1,70)$ & $1,55(1,54-1,59)$ & 0.035 \\
TAS after OPU & $1,66(1,58-1,68)$ & $1,58(1,57-1,62)$ & 0,010 \\
TOS before OPU & $1,66(1,58-1,68)$ & $1,60(1,54-1,62)$ & 0.092 \\
TOS after OPU & $1,63 \pm, 057$ & $1,58 \pm, 050$ & 0,013 \\
TAS before ET & $1,65 \pm, 072$ & $1,59 \pm, 054$ & 0,022 \\
TAS after ET & $1,68 \pm, 059$ & $1,62 \pm, 052$ & 0.010 \\
TOS before ET & $1,65 \pm, 063$ & $1,58 \pm, 062$ & 0.014 \\
TOS after ET & $1,62 \pm, 061$ & $1,57 \pm, 066$ & 0.065 \\
\hline
\end{tabular}

For normally distributed variables Mean \pm Standard Deviation (SD) values were given, for non-normally distributed variables median $(25 \%-75 \%)$ percentiles were given

Serum total anti-oxidant (TAS) in mmol Trolox Eq/L unit and serum total oxidant (TOS) in $\mu m_{0} \mathrm{H}_{2} \mathrm{O}_{2} \mathrm{Eq} / \mathrm{L}$ unit

$O P U$ oocyte pick-up, ET embryo transfer

$P$-value $<0.05$ was considered statistically significant 
Fig. 1 Serum total antioxidant status (TAS) levels in clinically pregnant and non-pregnant women around oocyte collection and embryo transfer

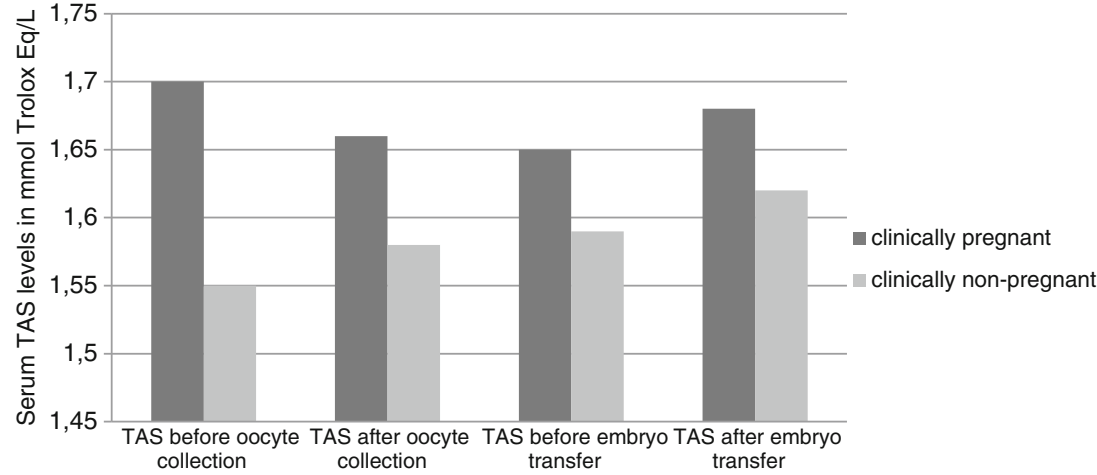

were evaluated with ROC curve analysis. According to power analysis calculations, the power of the study was $>80 \%$ for measurements of TAS before and after ET and TOS after oocyte retrieval. The sample size must be at least 14 in each group to obtain $80 \%$ power for TOS measurements before ET and at least 24 for TOS measurements after ET. A power analysis was performed for variables that were evaluated with parametric statistical tests.

\section{Results}

The cycle outcome and clinical data of 70 couples with primary infertility are summarized in Table 1 . No significant difference was noted between couples who had positive clinical pregnancy and those who did not in terms of the woman's age, duration of infertility, day 3 FSH-LHestradiol levels, day 3 AFC, number of retrieved oocytes, number of day 3 grade I+II embryos or number of transferred embryos.

Measurements obtained prior to OPU in patients with and without clinical pregnancy revealed median TOS levels of $1.66 \mu \mathrm{mol} \mathrm{H}_{2} \mathrm{O}_{2} \mathrm{Eq} / \mathrm{L}$ and $1.60 \mu \mathrm{mol} \mathrm{H}_{2} \mathrm{O}_{2}$ $\mathrm{Eq} / \mathrm{L}(P=0.092)$, respectively, and median TAS levels of $1.70 \mathrm{mmol}$ Trolox Eq/L and $1.55 \mathrm{mmol}$ Trolox Eq/L $(P=0.035)$, respectively. Measurements obtained after OPU in patients with and without clinical pregnancy revealed mean TOS levels of $1.63 \mu \mathrm{mol} \mathrm{H}_{2} \mathrm{O}_{2} \mathrm{Eq} / \mathrm{L}$ and $1.58 \mu \mathrm{mol} \mathrm{H}_{2} \mathrm{O}_{2} \mathrm{Eq} / \mathrm{L}(P=0.013)$, respectively, and median TAS levels of $1.66 \mathrm{mmol}$ Trolox Eq/L and $1.58 \mathrm{mmol}$ Trolox Eq/L $(P=0.010)$, respectively (Table 2$)$.

Prior to ET in patients with and without clinical pregnancy, the mean TOS levels were $1.65 \mu \mathrm{mol} \mathrm{H}_{2} \mathrm{O}_{2} \mathrm{Eq} / \mathrm{L}$ and $1.58 \mu \mathrm{mol} \mathrm{H}_{2} \mathrm{O}_{2} \mathrm{Eq} / \mathrm{L}(P=0.014)$, respectively, and the mean TAS levels were $1.65 \mathrm{mmol}$ Trolox $\mathrm{Eq} / \mathrm{L}$ and $1.59 \mathrm{mmol}$ Trolox Eq/L $(P=0.022)$, respectively. Measurements obtained after ET in patients with and without clinical pregnancy revealed mean TOS levels of $1.62 \mu \mathrm{mol} \mathrm{H}_{2} \mathrm{O}_{2} \mathrm{Eq} / \mathrm{L}$ and $1.57 \mu \mathrm{mol} \mathrm{H}_{2} \mathrm{O}_{2} \mathrm{Eq} / \mathrm{L}(P=0.065)$, respectively, and mean TAS levels of $1.68 \mathrm{mmol}$ Trolox Eq/L and $1.62 \mathrm{mmol}$ Trolox Eq/L $(P=0.010)$, respectively (Table 2$)$. The TAS levels in patients with clinical pregnancy were significantly higher prior to and after OPU and ET (Fig. 1, Table 2). In addition, TOS measurements after OPU and prior to ET were significantly higher in patients who had clinical pregnancy (Table 2). Comparison of TOS and TAS levels in different phases of $\mathrm{COH}$ cycles in clinically pregnant and non-pregnant patients were demonstrated in Tables 3 and 4.

According to OSI calculations it was found that; only the pre-OPU OSI was significantly lower in clinically pregnant patients (0.970) compared with non-pregnant ones (1.006) $(P=0.008)$ (Table 5$)$. Critical cut-off values of significantly different TAS and TOS levels, OSIes for clinical pregnancy were determined. They were $<0.98 \mathrm{mmol}$ Trolox Eq/ $\mu \mathrm{mol}$ $\mathrm{H}_{2} \mathrm{O}_{2}$ Eq with $\mathrm{AUC}=0.806(95 \% \mathrm{CI}, 0.617-0.928)$ and $p=0.001$ for OSI before OPU; $>1.6 \mathrm{mmol}$ Trolox Eq/L with $\mathrm{AUC}=0.747(95 \% \mathrm{CI}, 0.552-0.889)$ and $p=0.032$ for TAS before OPU; $>1.65 \mathrm{mmol}$ Trolox Eq/L with $\mathrm{AUC}=0.800$ $(95 \% \mathrm{CI}, 0.611-0.924)$ and $p<0.001$ for TAS after OPU; $>1.64 \mathrm{mmol}$ Trolox Eq/L with $\mathrm{AUC}=0.722$

Table 3 Comparison of TOS and TAS levels in different phases during $\mathrm{COH}$ cycles in clinically pregnant patients

Phases of cycle

\begin{tabular}{lll}
\hline & TOS levels & $P$ value \\
Before OPU-after OPU & $1,61 \pm, 061-1,63 \pm, 057$ & 0.014 \\
Before ET-after ET & $1,65 \pm, 063-1,62 \pm, 061$ & 0,003 \\
Before OPU-after ET & $1,61 \pm, 061-1,62 \pm, 061$ & 0.528 \\
& TAS levels & $P$ value \\
Before OPU-after OPU & $1,64 \pm, 075-1,64 \pm, 049$ & 0,831 \\
Before ET-after ET & $1,68(1,57-1,72)-1,71$ & 0.007 \\
Before OPU-after ET & $(1,63-1,74)$ & \\
\hline
\end{tabular}

For normally distributed variables Mean \pm Standard Deviation (SD) values were given, for non-normally distributed variables median (25\%-75\%) percentiles were given

Serum total anti-oxidant (TAS) in mmol Trolox Eq/L unit and serum total oxidant (TOS) in $\mu \mathrm{mol} \mathrm{H}_{2} \mathrm{O}_{2} \mathrm{Eq} / \mathrm{L}$ unit

$O P U$ oocyte pick-up, ET embryo transfer

$P$-value $<0.05$ was considered statistically significant 
Table 4 Comparison of TOS and TAS levels in different phases during $\mathrm{COH}$ cycles of clinically non-pregnant patients

\begin{tabular}{lll}
\hline TOS levels & & $P$ value \\
Before OPU-after OPU & $1,57 \pm, 046-1,58 \pm, 050$ & 0.778 \\
Before ET-after ET & $1,60(1,54-1,62)-1,58(1,52-1,60)$ & 0,020 \\
Before OPU-after ET & $1,57 \pm, 046-1,57 \pm, 066$ & 0.552 \\
TAS levels & & $P$ value \\
Before OPU-after OPU & $1,57 \pm, 053-1,59 \pm, 043$ & 0,017 \\
Before ET-after ET & $1,59 \pm, 054-1,62 \pm, 052$ & $<0.001$ \\
Before OPU-after ET & $1,57 \pm, 053-1,62 \pm, 052$ & $<0.001$ \\
\hline
\end{tabular}

For normally distributed variables Mean \pm Standard Deviation (SD) values were given, for non-normally distributed variables median (25\%-75\%) percentiles were given

Serum total anti-oxidant (TAS) in mmol Trolox Eq/L unit and serum total oxidant (TOS) in $\mu \mathrm{mol} \mathrm{H}_{2} \mathrm{O}_{2} \mathrm{Eq} / \mathrm{L}$ unit

$O P U$ oocyte pick-up, ET embryo transfer

$P$-value $<0.05$ was considered statistically significant

(95\%CI, $0.526-0.871)$ and $p=0.074$ for TAS before ET; $>1.67 \mathrm{mmol}$ Trolox Eq/L with AUC $=0.786(95 \% \mathrm{CI}$, $0.595-0.916)$ and $p=0.003$ for TAS after ET; $>1.64 \mu \mathrm{mol}$ $\mathrm{H}_{2} \mathrm{O}_{2}$ Eq/L with AUC $=0.758(95 \% \mathrm{CI}, 0.564-0.897)$ and $p=0.016$ for TOS after OPU; $>1.64 \mu \mathrm{mol} \mathrm{H}_{2} \mathrm{O}_{2} \mathrm{Eq} / \mathrm{L}$ with AUC $=0.761(95 \%$ CI, 0.567-0.899) and $p=0.017$ for TOS before ET. ROC analysis of significantly different seven measurements were shown in Figs. 2 and 3.

\section{Discussion}

Our study showed that the blood TAS level during OPU (pre and post) and ET (pre and post), most likely the most important phases in the ART-ICSI cycle, has a predictive value for clinical pregnancy. All measurements obtained before and after these important stages showed that TAS levels were significantly higher in patients who developed clinical pregnancy; the TAS levels before OPU were $1.70 \mathrm{mmol}$ Trolox Eq/L vs. $1.55 \mathrm{mmol}$ Trolox Eq/L $(P=0.035)$, respectively, and $1.66 \mathrm{mmol}$ Trolox $\mathrm{Eq} / \mathrm{L}$ vs. $1.58 \mathrm{mmol}$ Trolox Eq/L after OPU $(P=0.010)$, respectively.
Before ET, the TAS levels were $1.65 \mathrm{mmol}$ Trolox Eq/L vs. $1.59 \mathrm{mmol}$ Trolox $\mathrm{Eq} / \mathrm{L}(P=0.022)$ in patients with and without clinical pregnancy, respectively, and after ET, the TAS levels were $1.68 \mathrm{mmol}$ Trolox Eq/L and $1.62 \mathrm{mmol}$ Trolox Eq/L $(P=0.010)$, respectively.

Although oxidative stress has an impact on male infertility, the number of studies on female infertility has increased recently [2, 3]. In an animal model study, Duleba et al. [8] showed that oxidative stress negatively affected the development of theca-interstitial cells. In contrast, oxidants have been shown to play a role in folliculogenesis, follicular maturation and ovulation $[11,19,20]$. Thus, it is likely that oxidative stress may affect oocyte development and the development of embryos [2]. In a clinical study, Das et al. [7] found that ROS levels in follicular fluid measured by the chemiluminescence method have an impact on the development and quality of embryos; additionally, the oocyte fertilization capacity diminished as ROS increased in the follicular fluid of 78 couples who were admitted to IVF treatment because of tubal infertility.

Oyawoye et al. [14] measured the basal total antioxidant capacity (TAC) and decline of TAC in the follicular aspirates of 63 couples and 303 follicle aspirations with a ferric-reducing antioxidant power (FRAP) assay. Although it was interesting to note that the decline in TAC was lower when the oocytes were fertilized and higher in association with embryo viability, ROS may have different effects at different stages of embryo development. In our study, in addition to the high levels of TAS at all measured times in the clinically pregnant group, TOS levels after OPU and prior to ET were also higher in patients with clinical pregnancy. But according to OSI values only lower OSI before OPU was significant for predicting clinical pregnancy. Moreover the highest AUC value, from the seven different significant measurements [1) pre-OPU OSI, 2) TAS prior to OPU, 3) TAS after OPU, 4) TAS before ET, 5) TAS after ET, 6) TOS after OUP, 7) TOS before ET], was 0.806 and related to pre-OPU OSI (Figs. 2 and 3). So, high levels of TOS at certain measurement times support the idea that elevated antioxidant levels alone are not sufficient for the development of clinical pregnancy; ROS may affect different phases of embryo

Table 5 OSI in different phases during $\mathrm{COH}$ cycles in clinically pregnant and non-pregnant women

\begin{tabular}{llll}
\hline OSI & Clinically pregnant $(n=22)$ & Clinically non-pregnant $(n=48)$ & $P$ value \\
\hline Before OPU & $0,970(0,976-1,000)$ & $1,006(0,987-1,012)$ & 0.008 \\
After OPU & $1,000(0,984-1,008)$ & $0,990(0,976-1,009)$ & 0,555 \\
Before ET & $0,994(0,994-1,003)$ & $0,997(0,987-1,006)$ & 0.688 \\
After ET & $0,965(0,947-0,971)$ & $0,963(0,950-0,979)$ & 0,832 \\
\hline
\end{tabular}

Median $(25 \%-75 \%)$ percentiles were given

Differences were evaluated with Mann-Whitney $U$ test

$O P U$ oocyte pick-up, ET embryo transfer, $O S I$ oxidative stress index

$P$-value $<0.05$ was considered statistically significant 


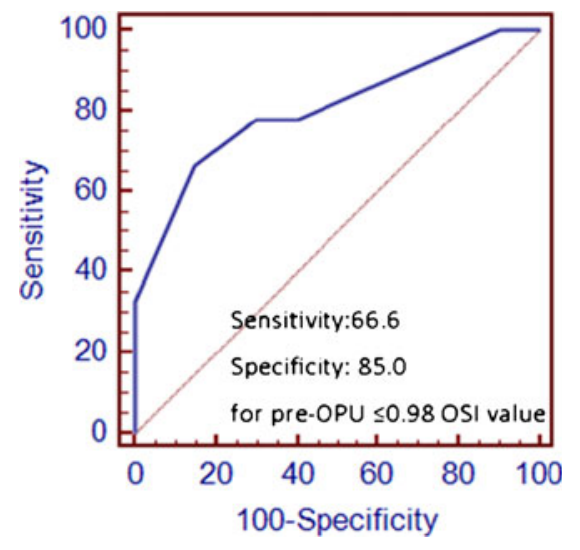

Fig. 2 ROC curve analysis for OSI before OPU. Sensitivity and specificity rates were given for pre-OPU OSI value which was significant for predicting clinical pregnancy and AUC was $0.806(95 \% \mathrm{CI}$, $0.617-0.928)$ with $p=0.001$. Pre-OPU OSI as in mmol Trolox Eq/ $\mu \mathrm{mol} \mathrm{H}_{2} \mathrm{O}_{2}$ Eq. (OPU; oocyte pick-up, OSI; oxidative stress index)

development, and the balance between oxidants and antioxidants may be as important as the antioxidant concentration.

In most of the studies on the role of ROS and antioxidants in ART treatment instead of clinical pregnancy, oocyte retrieval and/or fertilization and/or embryo development were considered as endpoints. Moreover, although isolated oxidative stress markers were used to determine ROS levels in some studies, total TOS measurement was performed in others [7, 11, 14]. Regarding TAS levels, isolated antioxidants were measured in some studies, while total TAS was measured in others [7, 14, 18]. Additionally, different methods were used for the measurements. These factors make it difficult to comment on how TAS and TOS affect clinical pregnancies because different substances were measured from different sites using different methods. In this study, we performed our measurements using the Erel method, which can determine the total antioxidant level to determine the TAS level [9]. We also measured TOS levels with the Erel method, which detects total oxidant levels instead of different oxidants [10]. In addition, measurements were obtained in female serum. To achieve clinical pregnancy, oocyte retrieval, fertilization and embryo development and endometrial implantation following ET are crucial. For this reason, we measured TAS and TOS levels only in the female serum, assuming that antioxidants and oxidants would be effective in all of the phases (oocyte development, embryo development and implantation). A unique facet of this study is that we determined the effect of serum TAS and TOS levels on predicting clinical pregnancy, which not only reflects folliculogenesis and embryogenesis but also systemic effects that may influence implantation.

In contrast, Oyawoye et al. [15] showed that the total antioxidant capacity depends on the etiology of infertility. The follicular total antioxidant capacity was higher in
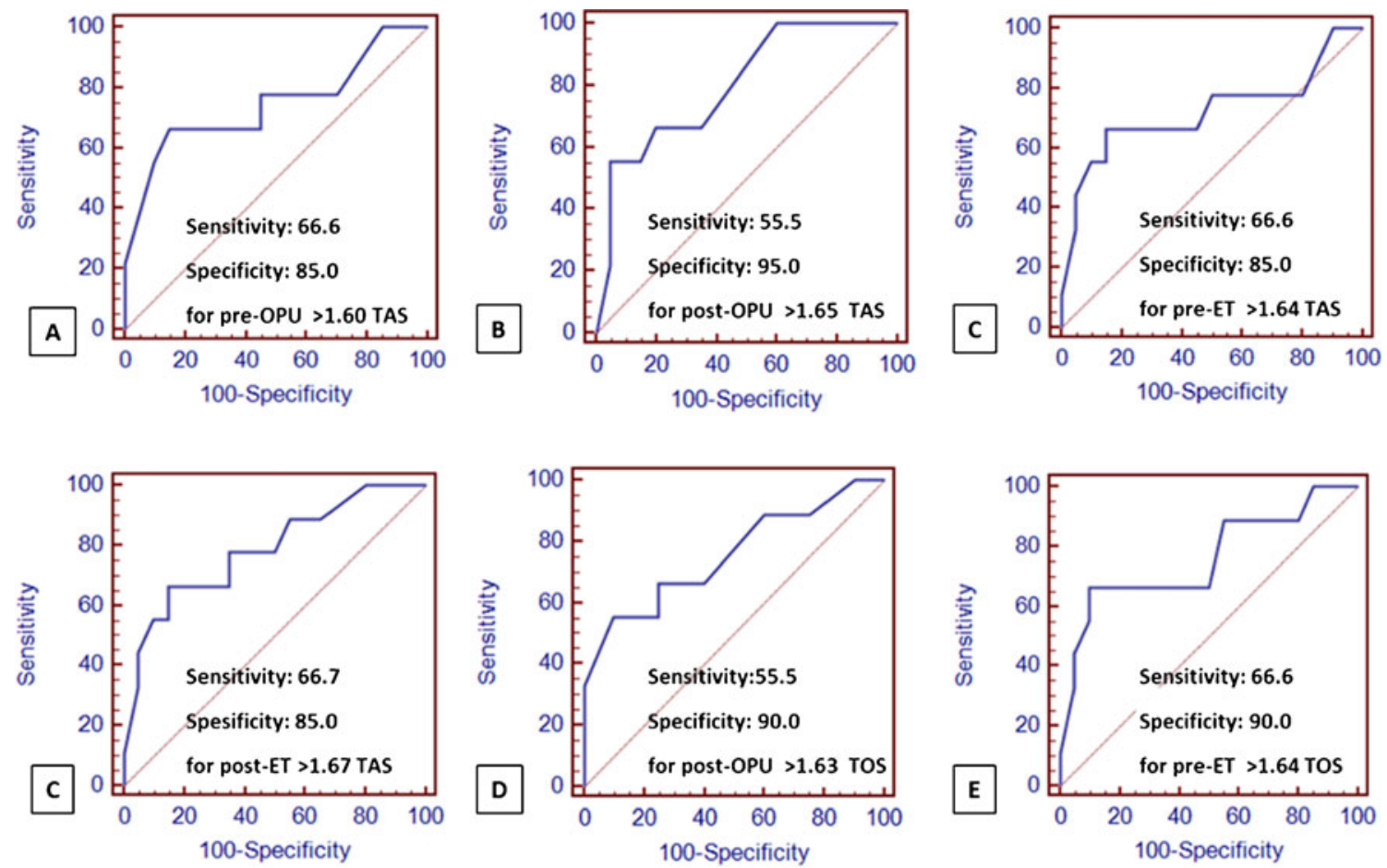

Fig. 3 ROC Curve analysis for measured variables that were significant for predicting clinical pregnancy. Significant critical cut-off values of TAS and TOS in different phases of $\mathrm{COH}$ cycle were given with sensitivity and specificity rates; a TAS prior to OPU, b TAS after OPU,

c TAS before ET, $\mathbf{d}$ TAS after ET, e TOS after OUP, $\mathbf{f}$ TOS before ET. Serum total anti-oxidant (TAS) in mmol Trolox Eq/L unit and serum total oxidant (TOS) in $\mu \mathrm{mol} \mathrm{H}_{2} \mathrm{O}_{2} \mathrm{Eq} / \mathrm{L}$ unit. OPU; oocyte pick-up, ET; embryo transfer 
patients with unexplained infertility and tubal factor infertility, while in patients with polycystic ovaries, an increase in total antioxidant capacity was related to fertilization incompetence. As Agarwal et al. [3] noted an imbalance between prooxidants and antioxidants can cause pathologies such as; endometriosis, PCOS, unexplained infertility and obstetric complications such as; abortion and preeclampsia. Therefore to improve the homogeneity of our study population, only infertile couples with male factor infertility were included in this study and most probably this is one of the most important limitations of our study. There is no suspicion about the effect of OS on male fertility, but our aim was to evaluate the effect of female OS on obtaining clinical pregnancy which develops after successful folliculogenesis, fertilization, embryogenesis and implantation. Studying with only male factor infertility couples is one of the most suitable designs that could show us the effect of female OS on clinical pregnancy. Additionally we also studied couples only with sperm can be obtained from ejaculation, so we totally excluded couples who had surgical sperm extraction or aspiration indication due to azoospermia. New studies including study groups of patient's exclusively with only one of the female infertility or male infertility etiology will improve our knowledge about this topic. Briefly; in an effort to induce the impact of oxidative stress induced by infertility etiology on our results minimal, we studied with couples who had only male factor infertility.

On the other hand, a study that was conducted with salivary stress biomarkers (cortisol and $\alpha$-amylase) showed that stress in the fertile window decreased the probability of conception [13]. The results of this study suggest that in couples that have similar infertility etiology, smoking status and age, high levels of serum TAS levels prior to and after OPU and ET increase the chance of pregnancy. Therefore, the amount of stress and the resultant imbalance between pro-oxidants and antioxidants in women during ART cycles has an impact on the occurrence of spontaneous pregnancy. In our study, we did not measure stress biomarkers, but due to the homogeneity of our study population, we believe that one of the most probable factors that affected the pregnancy rates and TAS/TOS levels is stress. Due to stress-related imbalances in oxidants and antioxidants, clinical pregnancy development was affected.

Currently, the trend is multivitamin and mineral supplementation in infertile patients in the aim of strengthening the antioxidant defense mechanism [16]. However, it seems obvious that we must strike a balance between antioxidants and oxidants instead of supplying infertile patients with antioxidant medications. Additionally, we must also attempt to decrease the stress of infertile patients.

One of the other limitations of the present study is that it is a prospective cohort study and not a randomized controlled trial; therefore, there may have been confounding variables influencing the results. However, we were able to show that the two study groups were comparable with respect to several parameters, including age, body mass index, and basal serum FSH levels, and therefore, the possibility of selection bias appear minimal. In contrast, none of the tests used for measuring TAS and/or TOS were demonstrated as the gold standard, and there is no consensus regarding which antioxidants or oxidants must be measured. However, we used novel Erel methods for both of the measurements.

In conclusion, in ART treatment cycles entirely indicated for male factor infertility, women with higher serum TAS levels in four major phases of $\mathrm{COH}$ cycles (pre-OPU, postOPU, pre-ET, post-ET) and with higher TOS levels preOPU and post-ET have an increased probability of clinical pregnancy. Additionally; OSI before OPU was one of the most important determinants for clinical pregnancy so the oxidant and antioxidant balance is as important as the antioxidant concentration alone.

\section{Acknowledgment None}

\section{References}

1. Agarwal A, Allamaneni SS. Role of free radicals in female reproductive diseases and assisted reproduction. Reprod BioMed Online. 2004;9:338-47.

2. Agarwal A, Gupta S, Sharma R. Oxidative stress and its implications in female infertility - a clinician's perspective. Reprod BioMed Online. 2005;11:641-50.

3. Agarwal A, Aponte-Mellado A, Premkumar BJ, Shaman A, Gupta $\mathrm{S}$. The effects of oxidative stress on female reproduction: a review. Reprod Biol Endocrinol. 2012;10:49-55.

4. Alpha Scientists in Reproductive Medicine and ESHRE Special Interest Group of Embryology. The Istanbul consensus workshop on embryo assessment: proceedings of an expert meeting. Hum Reprod. 2011;26:1270-83.

5. Bedaiwy MA, Falcone T, Mohamed MS, Aleem AN, Sharma R, Worley SE, et al. Differential growth of human embryos in vitro: role of reactive oxygen species. Fertil Steril. 2004;82:593-600.

6. Bedaiwy MA, Mahfouz RZ, Goldberg JM, Sharma R, Falcone T, Abdel Hafez MF, et al. Relationship of reactive oxygen species levels in day 3 culture media to the outcome of in vitro fertilization/ intracytoplasmic sperm injection cycles. Fertil Steril. 2010;94:2037-42.

7. Das S, Chattopadhyay R, Ghosh S, Ghosh S, Goswami SK, Chakravarty BN, et al. Reactive oxygen species level in follicular fluid-embryo quality marker in IVF? Hum Reprod. 2006;21:2403-7.

8. Duleba AJ, Foyouzi N, Karaca M, Pehlivan T, Kwintkiewicz J, Behrman HR. Proliferation of ovarian theca-interstitial cells is modulated by antioxidants and oxidative stress. Hum Reprod. 2004;19:1519-24.

9. Erel O. A new automated method to measure total antioxidant response against potent free radical reactions. Clin Biochem. 2004;37:112-9.

10. Erel O. A new automated colorimetric method for measuring total oxidant status. Clin Biochem. 2005;38:1103-11.

11. Jozwik M, Wolczynski S, Szamatowicz M. Oxidalive stres markers in preovulatory follicular fluid in humans. Mol Hum Reprod. 1999;5:409-13. 
12. Lee TH, Lee MS, Liu CH, Tsao HM, Huang CC, Yang YS. The association between micro environmental reactive oxygen species and embryo development in assisted reproduction technology cycles. Reprod Sci. 2012;19:725-32.

13. Louis GM, Lum KJ, Sundaram R, Chen Z, Kim S, Lynch CD, et al. Stress reduces conception probabilities across the fertile window: evidence in support of relaxation. Fertil Steril. 2011;95:2184-9.

14. Oyawoye O, Abdel Gadir A, Garner A, Constantinovici N, Perrett $\mathrm{C}$, Hardiman P. Antioxidants and reactive oxygen species in follicular fluid of women undergoing IVF: relationship to outcome. Hum Reprod. 2003;18:2270-4.

15. Oyawoye OA, Abdel-Gadir A, Garner A, Leonard AJ, Perrett C, Hardiman P. The interaction between follicular fluid total antioxidant capacity, infertility and early reproductive outcomes during in vitro fertilization. Redox Rep. 2009;14:205-13.

16. Ozkaya MO, Nazıroğlu M. Multivitamin and mineral supplementation modulates oxidative stress and antioxidant vitamin levels in serum and follicular fluid of women undergoing in vitro fertilization. Fertil Steril. 2010;94:2465-6.
17. Prieto L, Quesada JF, Cambero O, Pacheco A, Pellicer A, Codoceo $\mathrm{R}$, et al. Analysis of follicular fluid and serum markers of oxidative stress in women with infertility related to endometriosis. Fertil Steril. 2012;98:126-30.

18. Sabatini L, Wilson C, Lower A, Al-Shawaf T, Grudzinskas JG. Superoxide dismutase activity in human follicular fluid after controlled ovarian hyper stimulation in women undergoing in vitro fertilization. Fertil Steril. 1999;72:102734.

19. Sugino N, Takiguchi S, Ono M, Tamura H, Shimamura $K$, Nakamura Y, et al. Nitric oxide concentrations in the follicular fluid and apoptosis of granulosa cells in human follicles. Hum Reprod. 1996;11:2484-7.

20. Tatnate K, Sengoku K, Ishikawa M. The role of superoxide dismutase in the human ovary and fallopian tube. J Obstet Gynaecol. 1995;21:401-409.

21. World Health Organization. WHO laboratory manual for the examination and processing of human semen. 5th ed. Geneva: World Health Organization; 2010. 